\title{
COMPOUND AND COMPLEX ODONTOMA ASSOCIATED WITH ERUPTION DISTURBANCES AND ITS MANAGEMENT: REPORT OF TWO CASES
}

\author{
Dinesh Kumar', Gurnam Singh², Swati Kapoor ${ }^{3}$, Shobit Sethi ${ }^{4}$, Puneet Bajaj ${ }^{5}$, Sanjay Mittal ${ }^{6}$ \\ ${ }^{1}$ Associate Professor, Department of Oral \& Maxillofacial Surgery, Bhojia Dental College and Hospital, Himachal Pradesh, India \\ ${ }^{2}$ Professor \& H.O.D., Department of Oral \& Maxillofacial Surgery, Bhojia Dental College and Hospital, Himachal Pradesh, India \\ ${ }^{3}$ Senior Lecturer, Department of Oral \& Maxillofacial Surgery, Bhojia Dental College and Hospital, Himachal Pradesh, India \\ ${ }^{4}$ Post Graduate Student, Department of Oral \& Maxillofacial Surgery, Bhojia Dental College and Hospital, Himachal Pradesh, India \\ ${ }^{5}$ Professor \& H.O.D., Department of Oral \& Maxillofacial Surgery, Bhojia Dental College and Hospital, Himachal Pradesh, India \\ ${ }^{6}$ Professor \& H.O.D., Department of Oral \& Maxillofacial Surgery, Bhojia Dental College and Hospital, Himachal Pradesh, India
} \begin{abstract}
treatment and ensures better prognosis.
Keywords: Puption disturbances, Odontoma, Management

INTRODUCTION

In 1967 Paul Broca, was the first who used the term odontoma to describe all the odontogenic tumours. " Now more accurately odontomas are considered as hamartomas rather than true neoplasms. ${ }^{2}$ Odontomas are the most common type and constitute of about $22 \%$ of all odontogenic tumours of the jaws. ${ }^{3}$ These tumours are composed of enamel, dentin and variable amounts of cementum and pulp. They are slow-growing tumours Corresponding Author: Dinesh Kumar E-mail: dr_dinesh78@yahoo.com Received: $3^{\text {rd }}$ January 2017 Accepted: $20^{\text {th }}$ March 2017 Online: $20^{\text {th }}$ May 2017

showing non-aggressive behaviour. ${ }^{3,4}$ In odontome, organisation of odontogenic cells fails to reach a normal state of morpho differentiation hence the hard tissues are laid down in an abnormal
\end{abstract}

ABSTRACT

The most common type of odontogenic tumours is odontomas. Generally, they are benign and asymptomatic in nature. This paper describes two varieties of odontomas in two different cases. In first case a compound odontoma present in maxillary anterior region lead to over retention of primary teeth and non eruption of permanent teeth. Retained deciduous teeth and the lesion were removed and permanent teeth were allowed to erupt and orthodontic treatment was done. In 2nd case a complex odontoma was incidentally found when patient reported for extraction of mandibular third molar which was surgically excised. The results achieved indicate that the early diagnosis of odontomas allows the adoption of a less complex and expensive

pattern. According to the World Health Organization classification, Odontomas are subdivided into compound and complex types. Compound odontomas is composed of multiple, small tooth like structures known as denticles. In first case report a compound odontoma present in maxillary anterior region lead to over retention of primary teeth and non eruption of permanent teeth. Retained deciduous teeth and the lesion were removed and permanent teeth were allowed to erupt and orthodontic treatment was started. In complex odontome, the calcified dental tissues appear as an irregular mass bearing no morphologic similarity to even 
rudimentary teeth. ${ }^{5}$ In 2 nd case a complex odontoma was incidentally found when patient reported for extraction of mandibular third molar which was surgically excised. Compound odontomas are more common than the Complex variety and are in the ratio of 2:1. ${ }^{6}$ Compound odontomas are often associated with impacted adjacent permanent teeth. Odontomas are very rarely associated with the primary teeth and occur more often in the permanent dentition. ${ }^{7}$ They are often associated with eruption disturbances and their surgical removal represents the best therapeutic option.

\section{CASE REPORT 1}

A 14 year old female patient was referred to the Department of Oral and Maxillofacial Surgery by his orthodontist for the bulging in the left upper anterior region. The patient had no significant medical history and had not reported oral trauma or infections. Intraoral examination showed the presence of the primary left central and lateral incisors over the physiological period of exfoliation, missing permanent central and lateral incisors and upper left canine was placed buccally (Figure 1). Molar relationship was class I and skeletal class I pattern. A buccal \& palatal swelling of $1 \mathrm{x} 1 \mathrm{cms}$ in size in relation to 21,22 were observed (Figure 2). The swelling extended from mesial aspect of 11 to 23 regions. The overlying mucosa was of normal colour. On palpation the swelling was hard in consistency. Orthopantomogram revealed calcified masses of radiopaque tooth-like structures juxtaposed to the crown of upper left central and lateral incisor

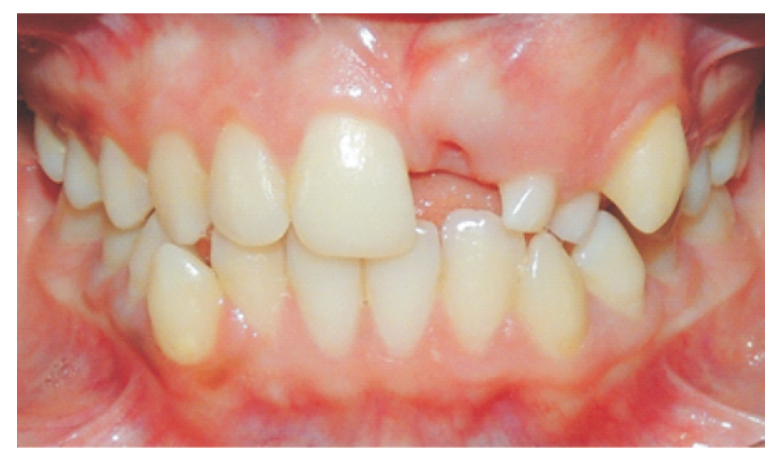

Figure1: Intraoral picture showing the presence of the retained primary left central and lateral incisors, missing permanent central and lateral incisors and upper left canine was placed buccally.

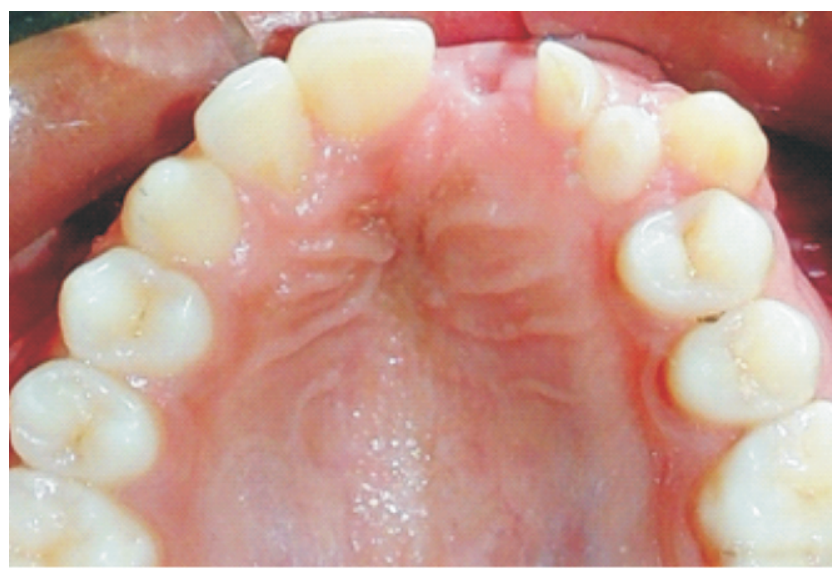

Figure 2: Buccal and palatal swelling was evident in anterior maxillary region

which was impacted near the nasal floor (Figure 3). Occlusal radiograph also showing a small mineralized mass in the left side of anterior maxillary region (Figure 4). The lesion was surgically exposed (Figure 5), removed (Figure 6) under local anaesthesia and closure was done with 3-0 mersilk suture (Figure 7).

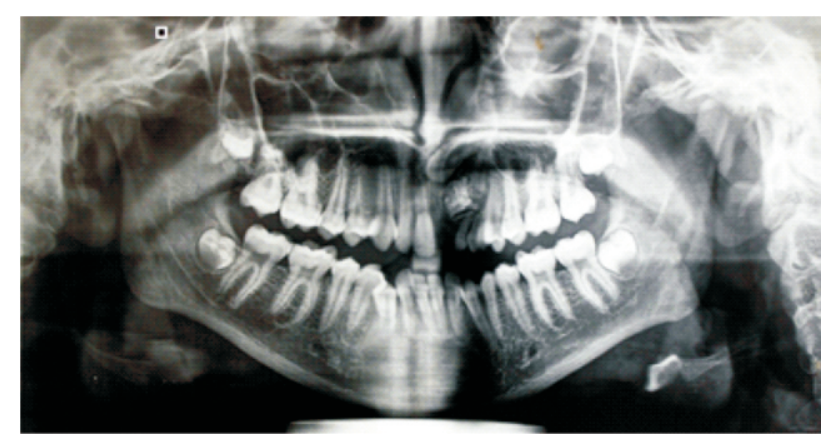

Figure 3: Pre-operative orthopantomogram showing the radiopaque mass in the left side of maxillary anterior region and impacted central and lateral incisors

Biopsy specimen was sent for histopathological examination to the department of oral pathology (Figure 8). Postoperative orthopantomogram show the complete removal of the lesion (Figure 9).

The gross findings showed a calcified mass consisting of multiple small teeth like denticles. The biopsy specimen was kept for decalcification. Histopathological report showed tooth-like structures made up of dentin, enamel matrix, pulp (Figure $10 \&$ 


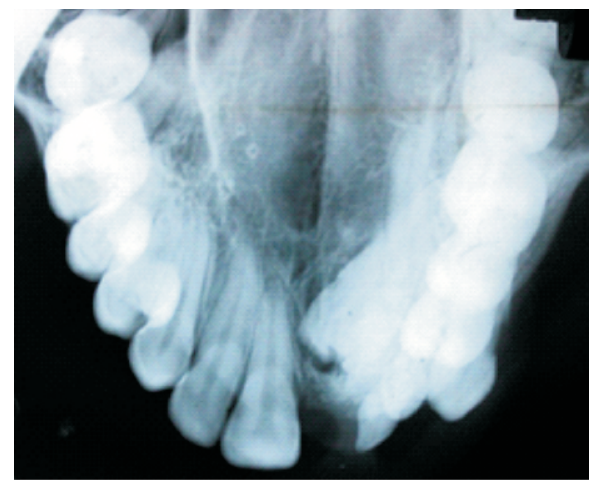

Figure 4: Occlusal radiograph showing small mineralized mass in the left side toward palatal region

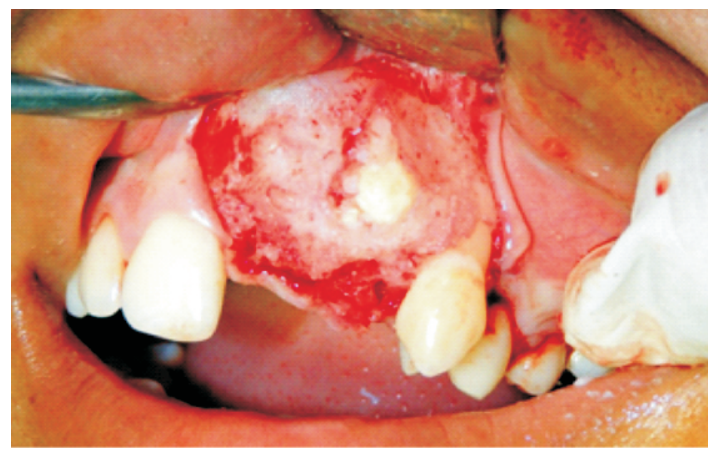

Figure 5: Surgical exposure of the lesion

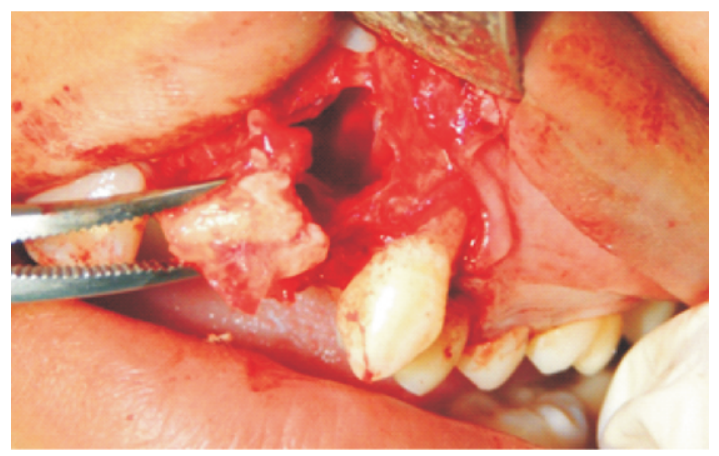

Figure 6: Surgical removal of the lesion

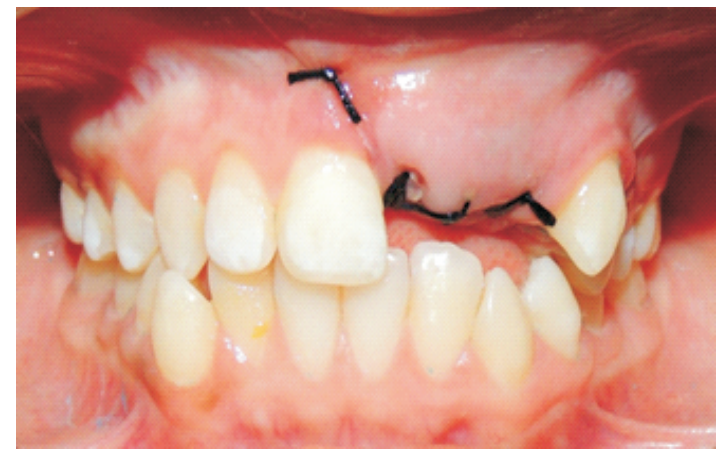

Figure 7: The flap repositioned and sutured

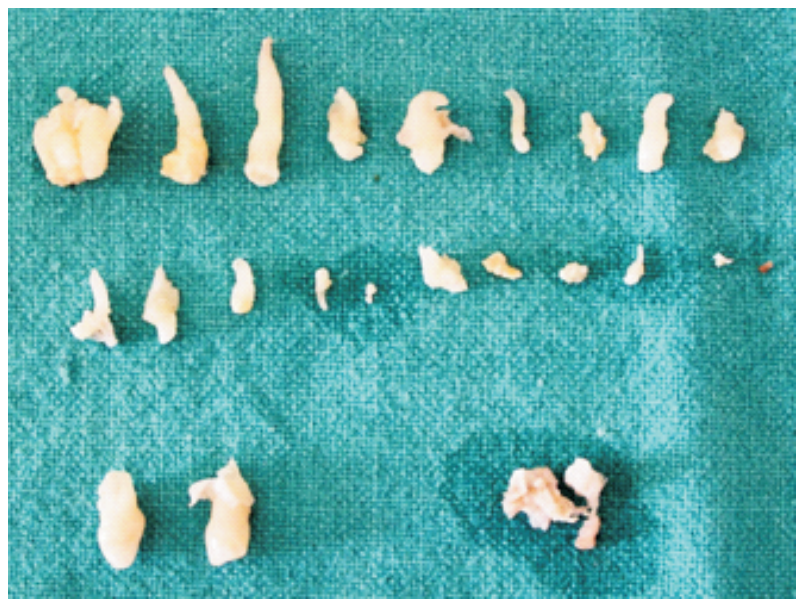

Figure 8: Ninteen mineralized structures with tooth-like appearance removed from interior of the lesion along with primary left central and lateral incisors

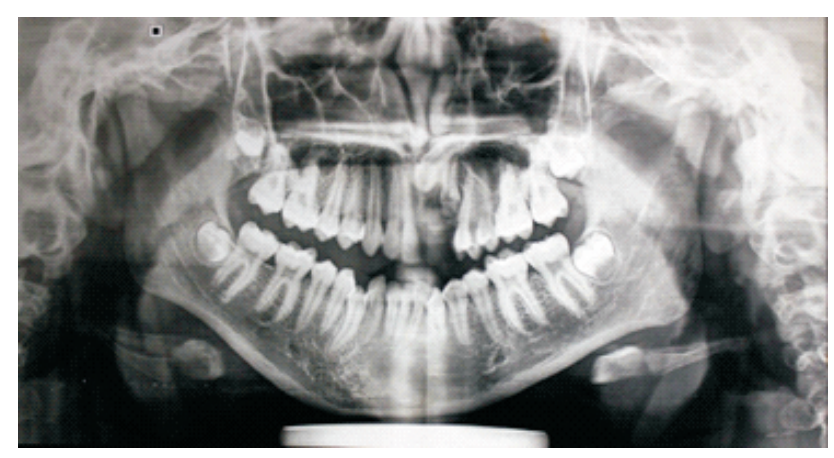

Figure 9: Post-operative orthopantomogram
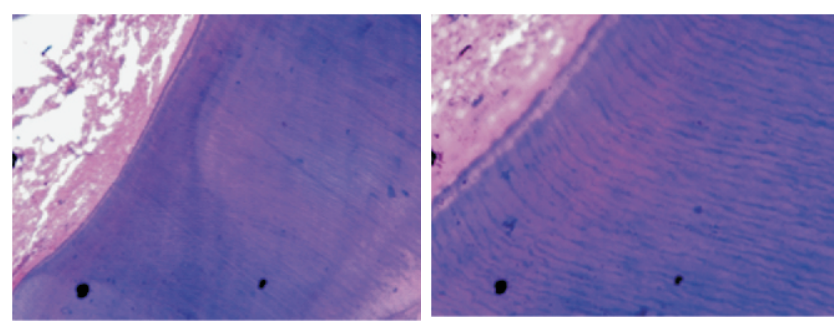

Figure 10 \& 11: Photomicrographs of the decalcified H\&E stained section show layers of dentine \& pulp like structures arranged in regular fashion. (10x and 40x)

11). Thus correlating the clinical, radiographic and microscopic findings, the diagnosis of compound odontoma was made. The postoperative period was uneventful. Postoperative treatment consisted of amoxicillin and clavulanic acid (625 $\mathrm{mg}$ thrice a day for 5 days), paracetamol (500mg twice a day for 3 days, and then as needed), and $0.2 \%$ chlorhexidine twice a day. 
The patient was referred to the orthodontist to continue the treatment. After two and half months, brackets were placed on impacted central and lateral incisors after surgical exposure of teeth (Figure 12).

On six months follow up, lateral incisor was fully erupted in the oral cavity and some space was being created for eruption of central incisor (Figure 13). On

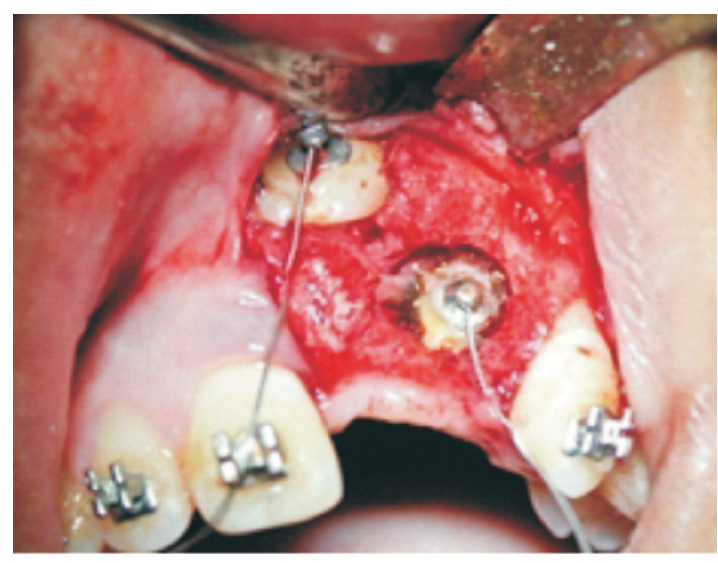

Figure 12: After two and half months, brackets were placed on impacted central and lateral incisors after surgical exposure

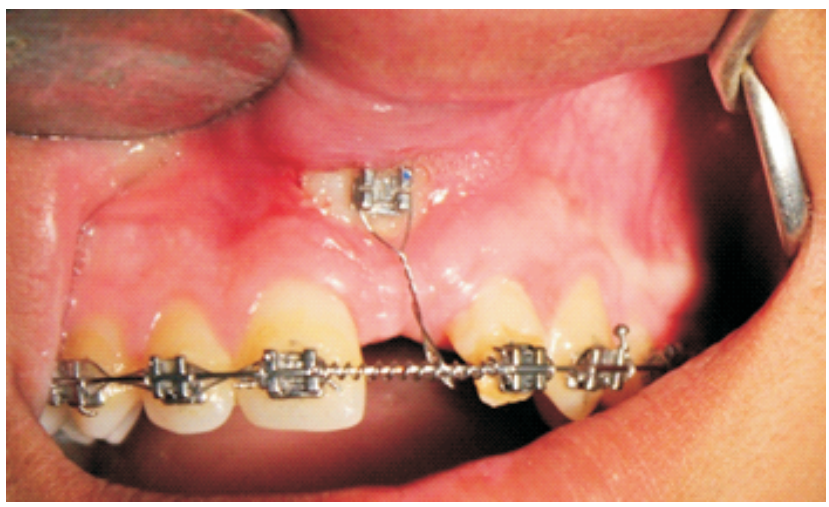

Figure 13: On six months follow up, lateral incisor was fully erupted in the oral cavity and some space was being created for eruption of central incisor.

18 months follow up, clinically and radiographically both incisors have erupted and are in normal occlusal position (Figure 14,15). Patient is still under orthodontic observation and final alignment of the teeth is being done.

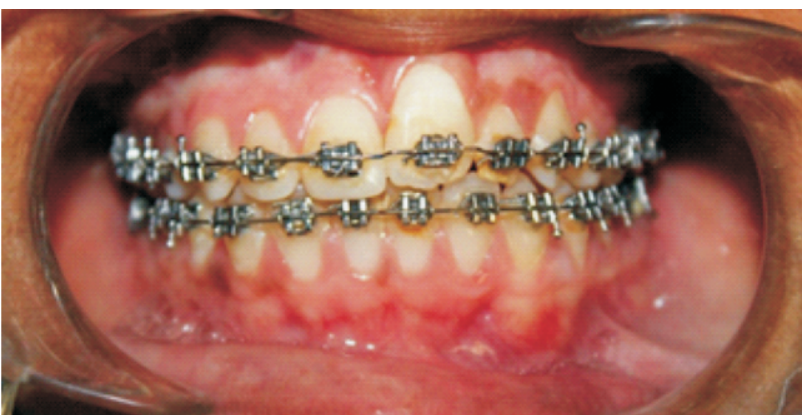

Figure 14: Post operative image showing complete eruption of central \& lateral incisor and come in occlusion after one year and six months

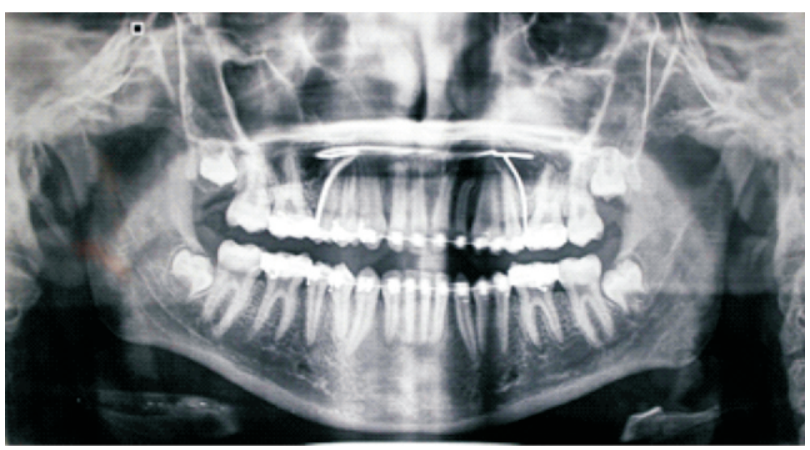

Figure 15: Post operative orthopantomogram image showing complete eruption of central and lateral incisor into the oral cavity after one year and six months

\section{CASE REPORT 2}

A 42-year-old patient referred to Department of Oral and Maxillofacial Surgery for extraction of right lower $3^{\text {rd }}$ molar of the jaw. On clinical examination, tooth was horizontally placed. An intraoral periapical (IOPA) radiograph was taken, which revealed a well defined homogenous opaque mass was seen between the roots of 46 and 48, covering the distal root of 46 and mesial root of 48 (Figure 16). The mass was covered with radiolucent halo, which was well appreciated on the mesial aspect. No secondary effects like resorption or displacement of the roots were seen. Based on both the clinical and radiological features, a provisional diagnosis of complex odontoma

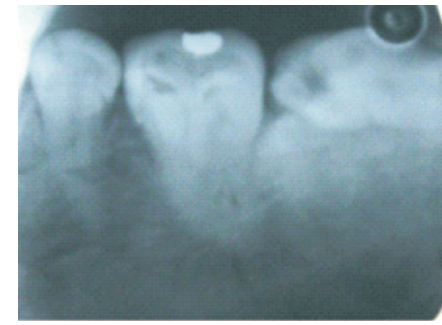

Figure 16: Intraoral periapical radiograph showing a well defined radiopaque mass between the roots of 46 and 48 
has been made. The mass was excised surgically (Figure 17) under local anaesthesia, and send for histopathologic examination. The biopsy report and histological slide confirmed the diagnosis of complex odontoma (Figure 18).

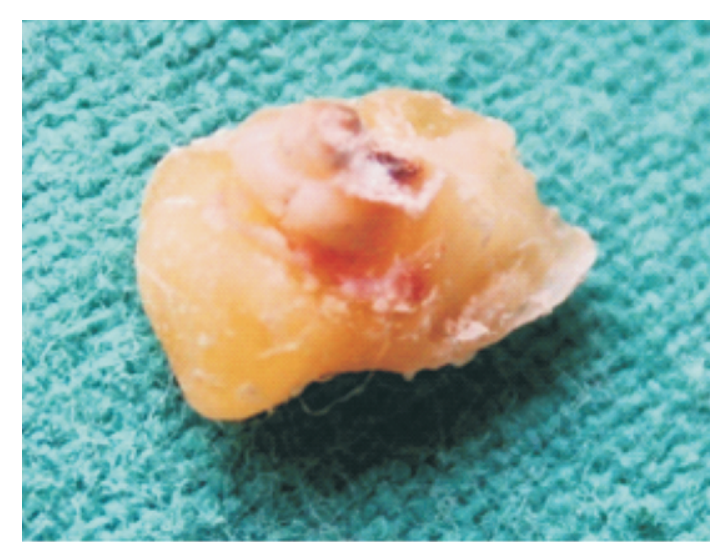

Figure 17: Excised mass of calcified material

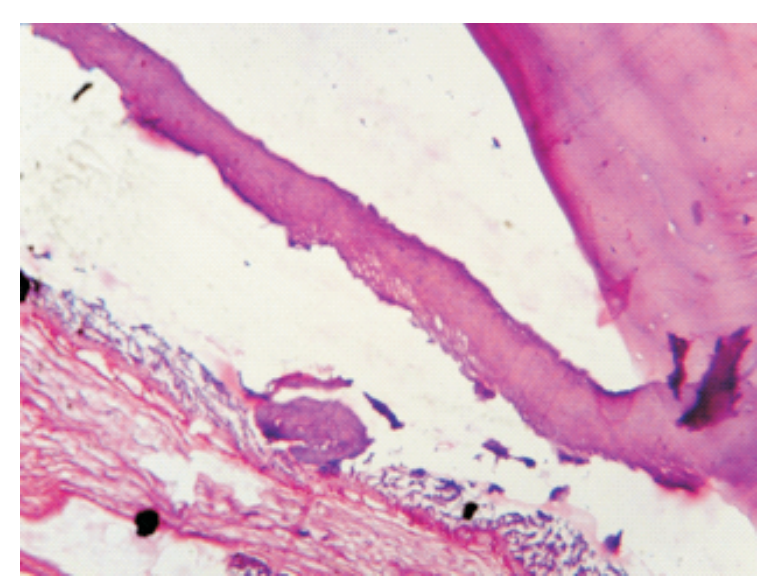

Figure 18: : Photomicrograph of the decalcified H\&E stained section shows irregular arrangement of enamel, enamel matrix, dentin and pulp like connective tissue. (10x)

\section{DISCUSSION}

The most common type of odontogenic benign jaws tumours are odontomas. The term odontoma is defined by Paul Broca as "tumours formed by the overgrowth of transitory or complete dental tissues. ${ }^{8}$ They are consisting of both epithelial and mesenchymal cells that present a complete dental tissue differentiation hence also called mixed tumours. ${ }^{9}$ The etiology of the odontome is largely unknown. It has been suggested that trauma and local infection may lead to the production of this lesion. Hitchin suggested that odontomas are either genetically inherited or are due to mutation in gene or post natal disturbance with genetic control of tooth development. ${ }^{5}$ In general, lesions are asymptomatic, slow growing and are usually discovered on routine radiographical examinations during the second and third decades of life. ${ }^{10}$

Odontomas can be found anywhere in the dental arches. The majority of compound odontoma has predilection toward the anterior region of the maxilla (61\%), whereas only $34 \%$ of complex odontomas occur in this area; while the complex odontomas shows a predilection for the posterior jaws (59\%) especially in the mandible and lastly the premolar area (7\%). Studies have shown that most of odontomes occur on right side of jaws, whereas in our first case odontome was seen in upper left quadrant ${ }^{11}$ and in $2^{\text {nd }}$ case, it was present in posterior region of right side in mandible. Our one patient was 14 year old female child and $2^{\text {nd }}$ patient was 42 year old female whereas average age of occurrence was found to be 20.3 years. ${ }^{12}$ Our both cases were female while there are controversy exists over gender distribution. Some studies show odontomas to be more common in females ${ }^{13}$ and others consider these lesions to be similarly distributed between both genders. ${ }^{14}$ Iatrous et al. ${ }^{15}$ and Yadav et al. ${ }^{16}$ found a male prediction in odontomas.

Clinically majority of odontomas are asymptomatic, although they rarely cause swelling, pain, suppuration, bony expansion, and displacement of teeth. ${ }^{6}$

It generally not exceed the size of a tooth, but when large can cause expansion of the cortical bone..$^{17,18}$ In our first case swelling was evident on labial as well as palatal side. Odontomas can vary in size from a few millimetres to many centimetres. ${ }^{19}$

According to the histopatological view, odontomas are classified as compound or complex: (a) compound /composite odontomas, in which their size and conformation are altered giving rise to multiple 
small tooth-like structures called denticles,${ }^{10}$ but dental tissues are normal. (b) Complex odontomas, exhibit a more or less disorderly arrangement but dental tissues are well formed. Odontomas may occur intraosseous or extraosseous. Intraosseous occur inside the bone and may erupt (erupted odontoma) into the oral cavity while peripheral or extraosseous occur in the soft tissue covering the tooth bearing portions of the jaws. ${ }^{20}$ In our reported cases intraosseous lesions were found.

The development of the odontoma is commonly associated with delayed exfoliation of primary teeth, impaction and eruption failure of permanent teeth. ${ }^{19-23}$ Radiographically, a developing odontoma are difficult in identification due to lack of calcification. It develop into three stages, in first stage absence of dental tissue calcification hence characterized by radiolucency, the intermediate or second stage shows partial calcification and the classically or third radiopaque stage exhibits surrounding radiolucent halo with the predominant tissue calcification. ${ }^{22}$ Compound odontoma shows calcified structures resembling teeth in the centre of a well-defined radiolucent lesion in first report 19 denticles were found. Complex type appears as an irregular mass of calcified material surrounded by a thin radiolucent area with smooth periphery. In second report a single irregular mass with maximum diameter of $1.0 \mathrm{~cm}$ was found. A pericoronary and periodontal space characteristic of unerupted teeth is seen around each tooth. ${ }^{23}$ In our first case, presence of odontoma prevented the physiological eruption of permanent maxillary central and lateral incisors of second quadrant also spaces seen around the teeth.

The histopathologically examination of odontomas often shows the presence of normal appearing enamel or enamel matrix, dentin, pulp tissue, and cementum which do not exhibit a normal relation to each other. ${ }^{1,7}$ Compound odontomas are formed by tooth-like structures which resemble pulp tissue in the central portion surrounded by a dentin shell and partially covered by enamel. Complex odontomas are conglomerates without orientation of dentin, enamel, enamel matrix, cementum, and areas of pulp tissue. The connective tissue capsule that surrounds an odontoma is similar to the follicle around a normal tooth. ${ }^{7}$ In $20 \%$ of the cases ghost cells are also seen. ${ }^{6}$ Histopathology reports of our cases confirmed the diagnosis of odontomas.

The treatment of choice for odontomas is surgical removal, followed by histopathological examination of biopsy to confirm the diagnosis. ${ }^{7,24-27}$ Literature review showed, the optimal management of the impacted tooth should allow its conservation and repositioning in the dental arch. ${ }^{24-26}$ In some cases, impacted teeth are frequently reported to be extracted simultaneously with the odontoma. ${ }^{27}$ These lesion have little tendency towards relapse hence prognosis is excellent.

\section{CONCLUSION}

Odontomas are benign tumours frequently seen in oral cavity. It is usually asymptomatic and associated with impacted, delayed permanent tooth eruption or temporary tooth displacement with or without a history of previous dental trauma. A careful knowledge and an excellent evaluation of $\mathrm{x}$-ray documents are essentials to resolve adequately each clinical case especially in pediatric patient. Early diagnosis and adoption of a conservative surgical approach is advisable to prevent later craniofacial complications, other developmental problems and ensures better prognosis.

\section{REFERENCES}

1. Baldawa R, Khante K, Kalburge J, Kasat V. Orthodontic management of an impacted maxillary incisor due to odontoma. Contemp Clin Dent 2011; 2:37-40.

2. Cawson R, Binnie W, Speight P, Barrett A, Wright J. Luca's pathology of tumors of the oral tissues, 1998; 83-5.

3. Neville BW, Damm DD, Allen CM, Bouquot JE. Oral and Maxillofacial pathology . Edn 2, Philadelphia WB Saunders 2002, 631-2.

4. Vengal M, Arora H, Ghosh S, Pai K. Large erupting complex odontoma: a case report. J Can Dent Assoc 2007; 73:169-72.

5. Rajendran R, Sivapathsundaram B. Shafer's textbook of Oral Pathology. Edn 5, Elsivier 2006, 405-406.

6. Cohen DM, Bhattacharyya I. Ameloblastic fibroma, 
ameloblastic fibroodontoma, and odontoma. Oral Maxillofac Surg Clin North Am 2004; 16(3):375-384.

7. Teruhisa U, Murakami J, Hisatomi M, Yanagi Y, Asaumi JA. case of unerupted lower primary second molar associated with compound odontoma. Open Dent J 2009; 3:173-176.

8. Gulsun YO, Gul T, Dilek K, Ercan D, Yagmur S. An unusual association of Odontomas with primary teeth. Eur J Dent 2007; 1:45-49.

9. Isler SC, Damircan S, Soluk M, Cebi Z. Radiological evaluation of an unusually sized complex odontoma involving the maxillary sinus by cone beam computed tomography. Quintissence International 2009;40(7):533-5.

10. Trivedi A, Gupta SD, Dua N, Mehta R, Mahajan S. Infected compound odontoma. A case report. BFUDJ 2010; 1(1) 63-5.

11. Shafer GW, Hine MK, Levy BM. A textbook of oral pathology (Edn 4) Philadelphia: WB Saunders 1983;308-11.

12. Slootweg PJ. An analysis of the interrelationship of the mixed odontogenic tumors: Ameloblastic fibroma, ameloblastic fibroodontoma and odontomas. Oral Surg 1981; 51:266-76.

13. Garcia-Consuegra L, Junquera LM, Albertos JM, Odontomas RO. A clinical-histological and retrospective epidemiological study of 46 cases. Med Oral 2000; 5:367-72.

14. An S, An C, Choi K. Odontoma: a retrospective study of 73 cases. Imaging Sci Dent 2012; 42:77-81.

15. Iatrous I, Vardas E, Theologie-Lygidakis N, Leventis M. A retrospective analysis of the characteristics, treatment and follow-up of 26 odontomas in Greek children. J Oral Sci 2010; 52:439-47.

16. Yadav M, Godge P, Meghana S, Kulkarni S. Compound odontoma. Contemp Clin Dent 2012; 3:S13-5.

17. Neville BW, Damm DD, Allen CM, Bouquot JE: Oral and Maxillofacial Pathology. Philadelphia: Saunders, 1995, pp 531-33.

18. Cawson RA, Binnie WH, Eveson JW: Color Atlas of Oral Disease. Clinical and Pathological Correlations. Hong Kong: Mosby-Wolfe, 1993, pp 6-19.
19. Singh S, Prerna, Uditi. Compound associated with an unerupted permanent lateral incisor. IJDS 2009; 1:9-14.

20. Wood NK, Goaz PW, Lehnert J. Mixed radiolucent-radiopaque lesions associated with teeth. In: Wood NK, Goaz PW, editors. Differential diagnosis of oral and maxillofacial lesions. Singapore: Harcourt Brace and Company Asia Pvt Ltd 1998, 289-314.

21. Junquera L, de Vincente JC, Roig P, Olay S, Rodriguez-Recio O. Intraosseous odontoma erupted into the oral cavity: an unusual pathology. Med Oral Patol Oral Cir Bucal 2005; 10(3):248-251.

22. Philipsen H, Reichart P, Praetorius F. Mixedodontogenic tumours and odontomas. Considerations on interrelationship. Review of the literature and presentation of 134 new cases of odontomas Oncol 1997; 32:86-99.

23. Owens BM, Schuman NJ, Mincer HH, Turner JE, Oliver FM: Dental odontomas: a retrospective study of 104 cases. J Clin Pediatr Dent 21:261-64, 1997.

24 M. Troeltzsch, J. Liedtke, V. Troeltzsch, R. Frankenberger, T. Steiner, and M. Troeltzsch, "Odontoma-associated tooth impaction: accurate diagnosis with simple methods? Case report and literature review," Journal of Oral and Maxillofacial Surgery, vol. 70, no. 10, pp. e516-e520, 2012.

25 M. Abdul, K. Pragati, and C. Yusuf, "Compound composite odontoma and its management," Case Reports in Dentistry, vol. 2014, Article ID107089, 4 pages, 2014.

26 K.S. Kannan, R. Prabhakar, R. Saravanan, Karthikeyan, and Rajvikram, "Composite compound odontoma-a case report," Journal of Clinical and Diagnostic Research, vol. 7, no. 10, pp. 2406-2407, 2013.

27 F. Angiero, S. Benedicenti, S. Parker, "Clinical and surgical management of odontoma," Photomedicine and Laser Surgery, vol. 32, no. 1, pp. 47-53, 2014.

Source of Support: Nil, Conflict of Interest: None Declared 